\section{Immune Globulins Don't Prevent Hepatitis C}

Postexposure prophylaxis following needlestick exposures to patients with hepatitis $\mathrm{C}$ virus infection has been the subject of numerous discussions in the infection control and public health community. Dr. K. Krawczynski and co-authors from the CDC's Hepatitis Branch, the Food and Drug Administration, and Chiron Corporation recently studied the efficacy of postexposure prophylaxis for the prevention of hepatitis $\mathrm{C}$ virus (HCV) infection in experimentally infected chimpanzees. Three chimpanzees were inoculated with $\mathrm{HCV}$; two were treated an hour later with anti-HCV-negative intravenous immune globulin (IGIV) or specially prepared hepatitis C immune globulin (HCIG), and the third animal was not treated. HCV infection was detected in all three animals within a few days of inoculation.

The overall results of this experiment indicated that neither HCIG or IGIV prevented or delayed HCV replication. Postexposure HCIG markedly prolonged the incubation period of acute hepatitis $\mathrm{C}$, but did not prevent or delay $\mathrm{HCV}$ infection. IGIV had no effect on the course of HCV infection. These results underscore the lack of efficacy of immune prophylaxis for healthcare workers following percutaneous exposures to $\mathrm{HCV}$-infected patients.

FROM: Krawczynski K, Alter M, Tankersley D, et al. Effective immune globulin on the prevention of experimental hepatitis C virus infection. J Infect Dis 1996;173:822-828.

\section{New Policy on Vancomycin}

Concern recently has been expressed by the CDC and the infection control community regarding a long-standing Healthcare Finance Administration (HCFA) policy regarding home intravenous antibiotics. Under the current policy, vancomycin is the only outpatient parenteral antibacterial agent that is reimbursed by Medicare. Consequently, an incentive has existed for the overuse of vancomycin, rather than use of other more appropriate antibiotics.

On March 5, 1996, HCFA, in a memo to its regional directors, indicated that, effective September 1, 1996, vancomycin no longer will be covered by Medicare. The use of an infusion pump for vancomycin was reviewed by HCFA because of a national public health concern related to the emergence of vancomycin-resistant enterococci in patient infections and from a review of their records that revealed a significant increase in home use, as measured by claims from the fourth quarter of 1994 to the third quarter of 1995.
HCFA concluded that an infusion pump is not necessary to administer the drug vancomycin in a safe and appropriate manner.

Medicare's policy of refusing reimbursement for outpatient parenteral antibiotic therapy has been an ongoing concern, because it contributes to prolonged in-patient lengths of stay to completed therapy and subsequent increased cost for Medicare. Healthcare experts argue that expansion of coverage would give doctors the ability to treat infections in less costly settings. These concerns have prompted the development of a House Bill, "Common Sense Medicare Parenteral Antibiotic Coverage Act of 1996," which is intended to expand Medicare coverage for parenteral antibiotic coverage.

FROM: HCFA. Memo to Regional Administrators Concerning Vancomycin. March 5, 1996.

\section{Hot Line}

The National Association of People with AIDS has launched a confidential, toll-free, 24-hour, cytomegalovirus (CMV) disease prevention hot line. The hot line is part of a broader CMV disease prevention and education program offered to healthcare professionals and people living with AIDS. The prevention and education program is sponsored, in part, by a grant from Roche Laboratories.

CMV can cause serious sight-threatening and lifethreatening illness in people who are immunocompromised. Approximately 40\% of people with HIV disease develop CMV retinitis, according the association. The CMV Hot Line number is 1-800-838-9990.

FROM: Voelker R. Quick uptakes: CMV hot line. JAMA 1996;275:901.

\section{Clinical Laboratory Self-Evaluations}

Health and Human Services Secretary Donna Shalala announced on April 3, 1996, that the Health Care Finance Administration (HCFA) has begun recertifying laboratories with exceptional past performance by allowing them to complete a self-survey questionnaire, the Alternate Quality Assessment Survey, in place of an onsite survey. To supplement the new survey process, no laboratory will go longer than 4 years without an onsite survey, and HCFA will perform random onsite surveys of a 5\% sample of laboratories that are using the new survey process to verify its effectiveness. The new system rewards laboratories with good performance and will allow HCFA to focus inspections better. 